\title{
Institucionalizar las políticas de inclusión digital: los programas de Argentina, Perú y Uruguay en perspectiva comparada
}

\author{
Damien Larrouqué
}

Sciences Po, París, Francia

Email: damien.larrouque@sciencespo.fr

\begin{abstract}
Resumen. Basado en una investigación de tesis doctoral, este artículo pretende explicar las razones de la institucionalización de los programas de inclusión digital, al comparar las modalidades de gestión e implementación de los planes Ceibal (Uruguay) y Conectar Igualdad (Argentina), todavía vigentes, con el proyecto Una Computadora por niño de Perú, abandonado a principio de los años 2010. Sostenemos que la preparación logística es determinante, como lo es también la arquitectura institucional. Concretamente, demostramos que los países que innovaron en materia administrativa, favoreciendo la participación de la sociedad civil y buscando mayor cooperación interinstitucional, lograron perennizar sus políticas públicas. Revelamos igualmente que el rol de la autoridad política (el presidente) es importante. Según nuestro postulado, estos programas son interesantes a estudiar porque ilustran el "regreso del Estado" en materia de inversión social, así como permiten apreciar su eficacidad a la hora de implementar políticas públicas presentadas como "revolucionarias".
\end{abstract} gentina

Palabras claves: Inclusión digital, política pública, Perú, Uruguay, Ar-

\section{Implementing digital inclusion policies in Latin America: the cases of Peru, Argentina and Uruguay}

\begin{abstract}
The ambition of this article, based on a doctoral research, is to bring into the light the factors of the institutionalization of digital inclusion policies, by comparing the modalities of management and implementation of Ceibal (Uruguay) and Conectar Igualdad (Argentina) programs with the Peruvian OLPC project. We argue that the quality of a policy design is key as well as the institutional structure. In order to successfully manage these public policies, we prove that the countries have to innovate in their administrative field, through the participation of civil society and a process of institutional cooperation. We also show that the role of the president (political authority) is important. Besides, as social issues, these programs can be considered as proofs of "the return of the state" and allow us to appreciate its ability to implement "revolutionary" public policies.
\end{abstract}

Keywords: digital inclusion, public policies, Peru, Uruguay, Argentina 


\section{Institucionalizar as politicas de inclusão digital: os programas de Peru, Argentina e Uruguai em perspectiva comparada}

Resumo. Baseado numa investigação de tese doutoral, este articulo pretende explicar as razões da institucionalização dos programas de inclusão digital, comparando as modalidades de gestão e implementação dos programas Ceibal (Uruguay) y Conectar Igualdad (Argentina), ainda vigentes, com o projeto Una Computadora por niño (Perú), abandonado a principio dos anos 2010. Sustentamos que a preparação logística é determinante, como o é também a arquitetura institucional. Concretamente, demostramos que os países que inovaram em matéria administrativa, favorecendo a participação da sociedade civil y procurando maior cooperação interinstitucional, conseguiram perenizar a suas políticas públicas. Igualmente, revalamos que o rol da autoridade política (o presidente) é importante. Conforme com nosso postulado, estes programas são interessantes para estudar porque ilustram o "regresso do Estado" em matéria de investimento social, e permitem apreciar a sua eficácia à hora de implementar políticas públicas apresentadas como "revolucionarias". gentina

Palavras chaves: inclusão digital, política pública, Peru, Uruguai, Ar-

\section{Introducción}

Inspiradas del proyecto norteamericano One laptop per child (OLPC), las políticas de alfabetización numérica o de inclusión digital consisten en la distribución de computadoras portátiles a alumnos escolarizados en establecimientos públicos. Esos programas son muy ambiciosos en la medida en que atañen varios sectores de intervención. Si el mejoramiento de la educación y el acceso universal al conocimiento son considerados objetivos prioritarios, esas políticas públicas también permiten favorecer la inclusión social de las poblaciones marginalizadas (vía el acceso generalizado a las herramientas informáticas), reforzar la integración territorial (vía el desarrollo de las infraestructuras de comunicación numérica) y hasta acelerar el desarrollo industrial (vía la valorización de la producción high tech local).

Desde nuestra perspectiva de investigación, más allá de ser "transpartidarias" - en el sentido de que interesan tanto a gobiernos de izquierda (Argentina, Uruguay, Venezuela) como de derecha (Colombia, México, Perú), las políticas de inclusión digital tienen una doble característica. Por una parte, son relativamente costosas en términos financieros - lo que supone un arbitraje político en su favor - y por otro lado, son muy exigentes de administrar al nivel logístico - lo que implica racionalizar las modalidades de gestión de la acción pública en países conocidos por su funcionamiento patrimonialista (Fernández Toro, 2009; Nef, 2003). En otras palabras, esos programas ilustran el "regreso del Estado" en materia de inversión social (Draibe y Riesco, 2007; Maillet, 2012; Rodriguez, 2010; Schweinheim, 2010; Thwaites Rey, 2008; Vallespín, 2010), así como permi- 
ten apreciar su eficacidad a la hora de implementar políticas publicas presentadas como "revolucionarias", o por lo menos, como "algo más que una computadora por niño” (Lamschtein y Rivoir, 2012).

Elegimos trabajar sobre tres políticas de inclusión digital. Por orden cronológico, se trata del plan Ceibal en Uruguay, anunciado por el presidente Tabaré Vázquez en diciembre del 2006, del plan Una Computadora Por Niño (UCPN) en Perú, presentado ante el Congreso por el ministro de Educación de Alan García, José Chang en octubre del 2007, y del plan Conectar Igualdad (CI) inaugurado por la presidenta Cristina Kirchner en Argentina en abril del 2010. Justificamos esos casos por el hecho de que constituyen, con el plan Cainama Educativo implementado en Venezuela a partir del 2009, las tres principales políticas de inclusión digital llevadas a cabo a escala nacional en América Latina. Además, desde el punto de vista analítico, son muy interesantes a estudiar en la medida en que unas se perennizaron y otra no.

En efecto, constatamos que a pesar de tener los mismos objetivos (lucha contra la brecha digital, revalorización de la educación, integración social), esas iniciativas de inclusión digital no obtuvieron los mismos resultados. Al superar las metas cifradas originales ${ }^{1}$, el plan Ceibal uruguayo y el plan CI argentino aparecen como auténticos éxitos políticos. Ambos resistieron a los trastornos electorales (cambio de gobierno Vázquez-Mujica en Uruguay y alternancia Kirchner-Macri en Argentina) y se institucionalizaron, al punto de ser considerados como un "programa estrella” en Uruguay (Lanzaro, 2010, p.64) y una “política de Estado” en Argentina (Lago Martínez et al., 2012, p.209).

Al contrario, en el caso peruano, el plan UCPN acumuló los problemas de implementación, como lo vamos a ilustrar más adelante. Entre 2007 y 2011, el ministerio de Educación del Perú (Minedu) compró apenas 900000 laptops a la fundación OLPC por un alumnado total estimado a unos 8 millones a nivel primario. Lo peor, es que estas laptops, por falta de capacitación, no han sido utilizadas por los docentes (Laura Quispe, 2015). Confrontado con las críticas ${ }^{2}$, acusado de haber permitido el enriquecimiento ilícito de una empresa vinculada a un ex senador aprista ${ }^{3}$, y evaluado de manera negativa por un equipo de investigadores independientes (Cristia et al. 2012) financiado por el Banco interamericano de desarrollo (BID), el plan UCPN ha sido abandonado después de la llegada al poder de Ollanta Humala en julio del 2011. Simbólicamente, el nuevo gobierno quitó la estatua del XO gigantesco (el modelo de laptop de OLPC) que había sido edificada ante el Minedu.

Basado en una investigación de tesis doctoral (Larrouqué, 2016), este artículo pretende explicar las razones de la institucionalización de los plan Ceibal y CI, al comparar sus modalidades de implementación con la del plan UCPN, considerado como un contraejemplo. Asimismo, el objetivo de la comparación no consiste en estudiar el proceso de difusión del concepto OLPC en América Latina, sino en analizar, a la luz de estas tres políticas de 
inclusión digital, las permanencias y evoluciones institucionales que impidieron o al contrario hicieron posibles sus implementaciones.

Después de haber aclarado las cuestiones metodológicas y precisado las dos principales hipótesis que estructuran esta reflexión, analizaremos esos tres programas de inclusión digital según dos secuencias: 1) la fase de policy design (entendido como el periodo de confección administrativa de una política pública) y 2) el proceso de implementación en sí (detallando el rol desempeñado por los diferentes actores institucionales). En nuestra conclusión, subrayaremos el rol del liderazgo presidencial, ya que no puede ser eludido a la hora de entender la institucionalización del plan Ceibal y CI.

\section{Consideración metodológica y presentación de las hipótesis}

Excluyendo el caso del proyecto OLPC Paraguay también estudiado en el marco de la tesis doctoral, se realizaron una quincena de entrevistas semiestructuradas por país, con jefes de programas, altos funcionarios, agentes administrativos, sindicalistas de la educación y profesores, entre enero y marzo 2011 (Montevideo), octubre y diciembre 2012 (Buenos Aires), y febrero y abril 2013 (Lima). Además, se realizó un trabajo sistemático de recolección de información a partir de los sitios internet en libre acceso de los principales periódicos de Argentina, Perú y Uruguay. En total, sin pretender a la exhaustividad, archivamos por fecha de publicación (20052015) alrededor de 150 artículos sobre el plan Ceibal, más de 100 sobre el plan CI y unos 40 respecto al plan UCPN.

Más allá del interés puramente factual, este trabajo archivístico nos permitió descartar la hipótesis según la cual el momento de la decisión política determinaría el “éxito” o "fracaso” de la acción pública. De hecho, como lo detallamos en otros trabajos (Larrouqué, 2017a y 2017b), que sea más o menos favorable, el contexto político-electoral del anuncio de los programas de inclusión digital no condiciona la buena o mala ejecución administrativa. Es verdad que el plan Ceibal ha sido presentado por Tabaré Vázquez en pleno "estado de gracia” (consecutivo al triunfo electoral de la izquierda por primera vez en la historia uruguaya y durante una fase de recuperación económica significativa) y se concluyó por un éxito políticoadministrativo; a diferencia del plan UCPN que emergió en un contexto particularmente adverso para el presidente García (exacerbación de la contestación social, después de su reelección "por defecto") y fue abandonado al cabo de cuatro años. Esos casos confirmarían la teoría de una influencia del contexto de la decisión sobre la implementación.

Sin embargo, el ejemplo argentino la invalida. De hecho, la presidente Cristina Kirchner lanzó el plan CI en un momento poco propicio: después que su partido haya perdido la mayoría legislativa en las elecciones anticipadas de junio 2009, poniendo la presidenta en una posición de lame duck. Para algunos politólogos, la presidenta no tenía "perspectivas creíbles de 
reelección” (De Luca y Malamud, 2010, p.173). En este contexto, el anuncio del plan CI fue muy criticado. La prensa de oposición lo aparentó a un programa demagógico destinado a seducir la clase media y a "sobornar" al electorado a través de la distribución de "pequeños regalos" informáticos ${ }^{4}$. Sin embargo, a pesar de esas críticas muy virulentas, el plan CI fue llevado a cabo y hasta proseguido por el presidente Macri. En breve, esos ejemplos nos confirman que el modelo de la ventana de oportunidad política (policy window) teorizado por Kingdon (1984) es solamente "probabilista” (Ravinet, 2010, p.282) y no permite inferir del "éxito” o "fracaso" de una política pública. El cuadro 1 sintetiza los elementos de contexto desarrollados en otros trabajos (Larrouqué, 2017a y 2017b)

\section{Cuadro 1 \\ El contexto político de la decisión en perspectiva comparada}

\begin{tabular}{|c|c|c|c|}
\hline & URUGUAY & PERÚ & ARGENTINA \\
\hline Presidente & $\begin{array}{c}\text { Tabaré Vázquez } \\
(2005-2010)\end{array}$ & $\begin{array}{l}\text { Alan Garcia } \\
(2006-2011)\end{array}$ & $\begin{array}{c}\text { Cristina Kirchner } \\
(2007-2015)\end{array}$ \\
\hline $\begin{array}{c}\text { Característica de la } \\
\text { victoria electoral }\end{array}$ & $\begin{array}{c}\text { Histórica } \\
\text { (izquierda en el poder } \\
\text { por primera vez) }\end{array}$ & $\begin{array}{c}\text { "Por defecto" } \\
\text { (elección del candidato } \\
\text { "menos peor") }\end{array}$ & $\begin{array}{c}\text { Sucesoria } \\
\text { (transferencia de la } \\
\text { popularidadde Nestor a } \\
\text { su esposa) }\end{array}$ \\
\hline $\begin{array}{l}\text { Configuración } \\
\text { parlamentaria }\end{array}$ & $\begin{array}{c}\text { Favorable } \\
\text { (Frente Amplio } \\
\text { mayoritario) }\end{array}$ & $\begin{array}{c}\text { Desfavorable } \\
\text { (APRA minoritaria) }\end{array}$ & $\begin{array}{c}\text { Variable } \\
\text { (FPV mayoritario hasta } \\
2009 \text { y después } \\
\text { minoritario) }\end{array}$ \\
\hline Coyuntura económica & En recuperación & $\begin{array}{c}\text { Muy buena } \\
\text { (pero crecimiento sin } \\
\text { inclusión) }\end{array}$ & Inestable \\
\hline Conflictividad social & Débil & Importante & $\begin{array}{l}\text { Creciente a partir de } \\
2008\end{array}$ \\
\hline "Estado de gracia" & $\begin{array}{l}\text { Largo y duradero } \\
\quad(2005-2008)\end{array}$ & Inexistente & $\begin{array}{c}\text { Diferido } \\
\text { (después de la muerte } \\
\text { de Nestor) }\end{array}$ \\
\hline $\begin{array}{l}\text { Anuncio del programa } \\
\text { de inclusión digital }\end{array}$ & Diciembre de 2006 & Octubre de 2007 & Abril de 2010 \\
\hline
\end{tabular}

Fuente: elaboración propia.

Para explicar por qué se institucionalizaron las políticas de inclusión digital de Uruguay y Argentina, mientras que la de Perú fue abandonada, consideramos dos hipótesis de corte neoinstitucionalista. Por una parte, la precipitación durante la fase de policy design genera distorsiones entre los medios y los fines (May, 2003) y, perjudica, in fine, el proceso de implementación. En el presente caso, la preparación logística del plan UCPN habría sido improvisada en comparación con la de los planes Ceibal y CI. Por otra parte, la gestión institucional de programas tan multidimensionales como lo son las políticas de inclusión digital necesitan alcanzar (o por lo menos tender a) la integración intersectorial (Cunill-Grau, 2014a). Como lo resume CunillGrau (2014b, p.7), "la resistencia a la colaboración interinstitucional constituyen uno de los factores claves de la brecha de implementación”. 
Concretamente, en el caso de Perú, el proyecto UCPN habría sido totalmente pensado, controlado y ejecutado por el propio ministerio de Educación (Minedu) cuyas debilidades institucionales son muy importantes (Balarin, 2005; Oliart, 2011), mientras que, en el caso del plan Ceibal y CI, los poderes públicos uruguayos y argentinos habrían buscado obtener la mejor sinergia interinstitucional posible, distribuyendo rol y compartiendo tareas ejecutivas, según las competencias, know-how o expertise de los diferentes organismos involucrados (públicos, parapúblicos y hasta privados). En suma, defendemos la tesis según la cual los programas de inclusión digital revelan una nueva forma de concebir y gestionar las políticas públicas en el Cono Sur, en el sentido de que se propicia más atención al policy design (o sea, más preparación logística) y se busca mejor ejecución institucional, al combatir los fenómenos contraproducentes de "insularidad administrativa” (Waissbluth, 2003).

\section{El policy design: la conversión de una decisión política en actos administrativos}

Aunque aceptamos la aserción de Michael Hill y Peter Hupe (2014) según la cual "la implementación es un proceso que debe ser distinguido de la formulación política”, sigue siendo muy difícil definir con precisión cuando se acaba la decisión política y cuando empieza la puesta en marcha administrativa. Inscribiéndose en la tradición analítica de Max Weber en Europa y de Woodrow Wilson en Estados Unidos, un primer enfoque teórico asumió, durante mucho tiempo, que existía una dicotomía fundamental entre la esfera política y la esfera administrativa. Redactado en 1970, el libro de análisis secuencial de Charles O. Jones (1984) es paroxístico de esta concepción a la vez segmentada y muy mecánica de la acción pública. A partir de los ochenta, iniciada por Mazmanian y Sabatier (1983), una nueva perspectiva de investigación se impuso. En vigor todavía, este enfoque considera que las etapas de la decisión, la formulación y la implementación son difícilmente disociables, en la medida en que se refuerzan y se entrelazan mutualmente.

Nuestra postura es intermediaria. A través del concepto de policy design, definimos la etapa de la formulación de la política pública como una fase de transición entre el momento de la decisión política y su ejecución administrativa. Consiste en la fase matricial del proceso de implementación. Es durante esta etapa que se define lo que un politólogo canadiense (Savard, 2010) denomina la cohesión interna (o “intracohesión”) de una política pública. Es decir la conversión de "su estado abstracto y general a su estado concreto y palpable” (Ibid, p. 331). El policy design moviliza algunos consejeros del gobierno y altos funcionarios, susceptibles de afirmarse como una "elite programática”" (Genieys, 2007). Esos actores son encargados de pensar las modalidades concretas de implementación, ya que deben traducir en actos administrativos los objetivos de la acción pública (efectividad), poner en sintonía los medios con los fines (eficacidad), elaborar los aspec- 
tos técnicos de la política pública (instrumentos), racionalizar sus costos (eficiencia), organizar idealmente la agenda administrativa en función del calendario electoral (timing), así como fijar las estrategias de despliegue (modalidades de extensión). En otras palabras, el policy design consiste en la matriz instrumental de una política pública. Sin policy design, la decisión política se queda meramente declarativa.

En esta sección que se divide en dos partes (consideraciones generales y aspectos técnicos), sostenemos que los países en los que el policy design ha sido muy cuidadoso han logrado institucionalizar sus políticas de inclusión digital, o sea Uruguay y Argentina. Para demostrarlo, pondremos en perspectiva la preparación logística, claramente expeditiva, del plan UCPN en Perú, en comparación con las del plan Ceibal y CI.

\section{Consideraciones generales: tiempo, objetivos, financiamiento y capital humano}

Primero, se puede comparar el periodo de gestación de los tres programas de inclusión digital para darse cuenta que el del plan UCPN ha sido mucho más corto que los del plan Ceibal y CI. En efecto, si tomamos como punto de partida el momento en el cual las autoridades políticas empezaron a interesarse en la iniciativa OLPC, y como fecha final el inicio de la distribución de las computadoras en las escuelas, se han tardado apenas 11 meses en el caso peruano (de julio 2006 a junio 2007). En el caso uruguayo, contamos 28 meses (de enero de 2005 a mayo de 2007) y casi 70 en el caso argentino (de julio 2005 a febrero 2011) ${ }^{6}$.

Además, los objetivos finales siempre aparecieron como confusos e indecisos en el Perú. A diferencia de los planes Ceibal y CI, el plan UCPN consistió en una simple tentativa de transposición a nivel nacional de un concepto internacional original (OLPC). En ausencia de una verdadera toma de posición del presidente García respecto a este proyecto, los ciudadanos peruanos en general y sobre todo los destinatarios del programa (alumnos, maestros, padres de familia) nunca supieron exactamente cuáles eran las finalidades exactas de tal política pública. Como prueba de una forma de improvisación, el Minedu modificó los alcances así como las modalidades de distribución apenas un año y medio después del lanzamiento del plan UCPN. Inicialmente destinadas a los alumnos de las escuelas rurales unidocentes bajo la modelo "uno a uno" de OLPC (una computadora, un niño), las computadoras (XO) fueron distribuidas a partir de octubre de 2009 en Centros de recursos tecnológicos, o sea en laboratorios informáticos dentro de los establecimientos públicos. Asimismo, más allá de duplicar iniciativas similares existentes en ciertas regiones o municipios, se “desvirtuó” el plan UCPN al no respetar al primero de los cincos fundamentos de OLPC: la computadora pertenece al niño y no a la escuela (child ownership). En los demás países, esta característica fundamental fue conservada, pero condicionada a la asistencia en clase, lo que en Argentina permitió reducir la deserción o el abandono escolar (Villanueva et al. 2011). En pocas palabras, en Perú, no se tomó el tiempo para precisar los objetivos y adecuarlos con las necesidades. 
Como pasó en Uruguay con los fondos extraordinarios del ministerio de Hacienda y en Argentina con las reservas de la Administración nacional de la seguridad social (ANSES), la cuestión del financiamiento no se planteó tampoco en Perú. El Minedu recibió un cheque en blanco por parte del Parlamento unicameral en octubre de 2007 para comprar las 40000 primeras maquinitas. Luego, cada año, en diciembre, una línea presupuestaria fue añadida a la ley del presupuesto para permitir que el Minedu comprara más laptops. Sin embargo, a diferencia de los países del estuario de la plata, estas compras se hicieron sin licitación internacional. En total, 900000 XO fueron compradas exclusivamente a la fundación OLPC, bajo pretexto de que iban a ser necesariamente más baratas que las laptops mercantiles. Si es cierto, los XO no equivalen tampoco a netbooks comerciales. Así que no se interrogó la eficiencia económica del proyecto. En Perú, se invirtieron unos 200 millones de dólares por casi un millón de computadoras que no fueron usadas (Laura Quispe, 2015), mientras que en Argentina se compró 5 millones de computadoras de mucha mejor calidad por un costo unitario estimado a unos 450 dólares (Diaz Rato y Fontdevlia, 2009, p.87), las cuales suscitaron mucho más entusiasmo por parte del cuerpo docente (Villanueva et al. 2011). En resumen, Perú no consideró la relación costo-beneficio de la política pública.

En comparación con los demás países, el gobierno de Perú tampoco invirtió suficientemente en capital humano. Se estima que unos 30 funcionarios dependiendo de la Dirección general de las tecnologías educativas (DIGETE) del Minedu trabajaron sobre el plan UCPN. En Uruguay, en el Laboratorio tecnológico de Uruguay (LATU), ente parapúblico rector del plan Ceibal, alrededor de 200 agentes fueron contratados a propósito. En cuanto a Argentina, además de haber reforzado su planta permanente, ANSES contrató a unos 1000 jóvenes para configurar cada computadora, una por una, instalando los diferentes softwares y programas educativos. Así, el gobierno argentino fue capaz de distribuir algunas 360000 netbooks en solamente un mes (septiembre 2011), o sea la mitad de lo que hizo el Minedu en cuatro años.

\section{Aspectos técnicos de la preparación logística}

Como el plan Huascarán y otras políticas de alfabetización numérica anteriores (Balarin, 2013), el plan UCPN es sintomático de una débil preparación político-administrativa en el Perú. Asimismo, la fase de prueba fue puramente formal. Concretamente, el proyecto-piloto de Arahuay (julio 2007) sirvió solamente para corregir los aspectos más problemáticos de las laptops (traducción de los softwares al español). En Uruguay al contrario, el proyecto-piloto de Cardal (mayo 2007) sirvió para remediar las imperfecciones técnicas de las XO (problemas de acceso a internet, conexión entre XO, etc.). Concretamente, la Comisión interinstitucional encargada del policy design del plan Ceibal (Comisión de políticas) exigió a la fundación OLPC mejorar las capacidades técnicas de sus laptops (Comisión de políticas, 2009). 
Además, en Perú, los desafíos logísticos e institucionales han sido subestimados y mal evaluados desde el principio. Como mínimo, la implementación de una política de inclusión digital exige asegurarse que los registros escolares sean fiables y que el conjunto de las escuelas disponen de un cierto número de requisitos, incluyendo por lo menos el acceso a la electricidad. Al Minedu, no parece haberle importado mucho la poca fiabilidad de sus registros institucionales, ni tampoco las deficiencias de las infraestructuras escolares. Por consiguiente, se distribuyeron más computadoras de las necesarias en algunas escuelas y menos en otras, así como en establecimientos sin energía eléctrica (Quispe y Diaz, 2010). Peor todavía, a diferencia de Uruguay, se acusó al gobierno peruano de haber regalado computadoras a alumnos que no tenían ni que comer (Oppenheimer, 2010).

En Argentina, país federal afectado por una enorme fragmentación institucional del sistema educativo (Rivas et al., 2004), el ministerio de Educación de la Nación no tenía tampoco a disposición registros fiables. Entonces, lo que decidió ANSES, el organismo rector del plan CI, fue contabilizar el número exacto de alumnos, atribuyéndolos por anticipación el código único de identificación profesional (CUIL) reservado para los mayores de edad. Vía un portal informático bautizado ConIg, se exigió a los directores de los 7000 establecimientos públicos considerados (colegios, liceos, escuelas normales e institutos especializados) que realizaran ellos mismos el registro informático de todos los alumnos, con la ayuda eventual de la sucursal de ANSES zonal. Este dispositivo permitió atribuir exactamente una computadora a cada alumno, centralizar toda la información e in fine racionalizar el proceso de distribución. Al contrario en Perú, no se organizó de manera rigurosa la distribución y no hubo ninguna planificación estratégica como en Uruguay. En este país de hecho, los responsables del plan Ceibal eligieron invertir las lógicas tradicionales de difusión de la acción pública, empezando la distribución de las computadoras por el Interior. Como lo explicamos anteriormente (Larrouqué, 2013), el objetivo no era solamente simbólico (revalorizar las escuelas rurales), sino político (cortocircuitar la oposición de los sindicatos de la enseñanza y vencer las reservas inicialmente muy fuertes del cuerpo docente).

En lo que concierne la capacitación de los docentes, Argentina es claramente el país que le consagró una mayor atención. Con el apoyo de la Organización de los Estados Iberoamericanos (OEI), el ministerio de Educación de la Nación (MEN) propuso una formación virtual de 12 semanas doce veces más que en Perú (40 horas), a la cual participaron unos 60000 profesores, o sea alrededor de un $20 \%$ del cuerpo docente (Diaz Rato y Fontdevlia, 2009, p.91-94). En cuanto a Uruguay, como el anuncio del plan Ceibal generó hostilidad por parte de la Administración Nacional de Educación Pública (ANEP), ente autónomo controlado por los docentes, la prioridad durante el policy design se orientó hacia la organización de la distribución y conexión de las computadoras. Las cuestiones didácticas fueron tratadas más tarde, después de que el ministerio de Educación y Cultura (MEC) y la ANEP empezaron a mejor coordinar sus acciones (Barboza Norbis, 2012). 
Finalmente, como lo resume el Cuadro 2, Perú aparece claramente como el país que menos tiempo, recursos humanos y esmero consagró a esta fase matricial de la acción pública.

\section{Cuadro 2}

\section{Las características comparadas del policy design de los programas de inclusión digital}

\begin{tabular}{|c|c|c|c|}
\hline & PERÚ & URUGUAY & ARGENTINA \\
\hline Nombre del programa & Plan UCPN & plan Ceibal & Plan Conectar Igualdad \\
\hline Duración en meses & 11 & 28 & 70 \\
\hline Agentes involucrados & Unos 30 & Unos 200 & Más de 1000 \\
\hline Licitación internacional & No & $\mathrm{Si}$ & $\mathrm{Si}$ \\
\hline Formación docente & Insuficiente & Tardía & Notable \\
\hline Planificación estratégica & No & $\mathrm{Si}$ & $\mathrm{Si}$ \\
\hline Cambios de modalidades & $\mathrm{Si}$ & No & No \\
\hline $\begin{array}{l}\text { Hipótesis preliminar en } \\
\text { cuanto al resultado }\end{array}$ & $\begin{array}{c}\text { Más susceptible de } \\
\text { abandono }\end{array}$ & $\begin{array}{l}\text { Más susceptible de } \\
\text { institucionalización }\end{array}$ & $\begin{array}{l}\text { Más susceptible de } \\
\text { institucionalización }\end{array}$ \\
\hline
\end{tabular}

Fuente: elaboración propia

Sin embargo, sería muy reductor confiar solamente en la calidad aparente del policy design (o sea en la preparación logística) para inferir del éxito de la política pública. Lo más importante consiste en la articulación entre policy design y ejecución administrativa. Como lo resumieron Pressman y Wildavsky (1981, P.XXV), "la separación entre la formulación de la política y su implementación es fatal”. En la siguiente parte, nos interesamos precisamente a las modalidades institucionales que prevalecieron en el proceso de implementación de estas tres políticas de inclusión digital.

\section{La implementación: en busca de la integración intersectorial}

La implementación constituye el corazón de la acción pública. Es la etapa durante la cual se determina el "éxito" o el "fracaso" relativo de una política pública. Como en otras partes del mundo, en América Latina, se tiende a considerar que consiste en una prueba de fuego para el gobierno, en la medida en que es a través de la acción pública que forja su credibilidad o, al contrario, la arruina (Agosta et al., 2012; Romero Quintero, 2013). Desde el punto de vista académico, existen dos principales enfoques para analizar los procesos de implementación. Inaugurada en Estados Unidos por los trabajos de Pressman y Wildavsky (1984) y en Europa por los reflexiones de Bruno Jobert y Pierre Muller (1987), la concepción top-down considera que los actores principales de las políticas públicas son las elites político-administrativas que las formulan.

$\mathrm{Al}$ contrario, los partidarios del enfoque inverso bottom-up o street level bureaucracy, cuyo inspirador es Michael Lipsky (2010), estiman que 
“el trabajo de los que unos llaman los agentes de ejecución se reduce raramente a una simple y estricta aplicación de las reglas e instrucciones nunca suficientemente unívocas para impedir la posibilidad de reinterpretación, ni suficientemente completas para dispensarse de adaptaciones a los casos particulares" (Dubois, 2010, p.174). Como ya lo vimos, nuestra postura es claramente top down. Sin embargo, adherimos a la tentativa de cotejo teórico propuesto por Richard Matland (1995). Según el universitario norteamericano, existen cuatro modelos ideal-típicos de implementación, los cuales son condicionados por dos factores: por una parte, la ambigüedad de la política pública (policy ambiguity) y, por otra parte, las tensiones (policy conflict) que la política pública es susceptible de generar. La ambigüedad política se refiere a la falta de claridad en los objetivos y/o en los medios para alcanzarlos (fallos en el policy design).

En cuanto al conflicto político - que es en realidad de carácter institucional, se refiere, en substancia, a la diferencia de visiones entre instituciones que, al no compartir la misma concepción de la acción pública, no esperan los mismos resultados. En ausencia de ambigüedad política y de rivalidades institucionales, como fue el caso en Argentina, se podría hablar de una implementación administrativa (administrative implementation). Se caracteriza por un proceso top-down clásico en el cual la autoridad política (el principal) confía a la administración (el agente) la misión de ejecutar sus resoluciones programáticas. En el caso de una débil ambigüedad política pero de fuertes divergencias institucionales, como se observaron en Uruguay con la oposición inicial de los docentes al plan Ceibal, la implementación sería calificada de política (political implementación). Esta modalidad implica una capacidad gubernamental de regateo, de coacción o de contorneo respecto a la administración (lógica by-pass).

La combinación de una fuerte ambigüedad política con débiles tensiones institucionales -tal cual lo constatamos en Perú - desembocaría en una implementación de carácter experimental (experimental implementation). El éxito de la política pública depende en este caso de la capacidad de los agentes administrativos en interpretar las voluntades gubernamentales y en apropiárselas. Por fin, la combinación de una fuerte ambigüedad política con fuertes disensiones institucionales - como lo fue el caso del proyecto OLPC de Caacupé en Paraguay - conduciría a una implementación puramente simbólica (symbolic implementation). En esas condiciones, la política pública tiene fuerte riesgos de quedarse simplemente en una declaratoria. De esos cuatros modelos, los más susceptibles de contribuir a la institucionalización de la política pública son los dos primeros (administrative y political implementation), tal cual lo resumimos en el Cuadro 3. 


\section{Cuadro 3 \\ Los modelos ideotípicos de Matland (1995) aplicados a las políticas de inclusión digital}

\begin{tabular}{|l|c|c|c|c|}
\hline \multicolumn{2}{|c|}{} & \multicolumn{2}{|c|}{ Ambigüedad de la política pública } & Probabilidad de \\
\cline { 3 - 5 } \multicolumn{2}{|c|}{} & Débil & Fuerte & Fuerte \\
\hline $\begin{array}{l}\text { Tensiones } \\
\text { institucionales }\end{array}$ & Débil & $\begin{array}{c}\text { Administative implementation } \\
\text { (plan CI en Argentina) }\end{array}$ & $\begin{array}{c}\text { Experimental implementation } \\
\text { (plan Ceibal en Uruguay) }\end{array}$ & Débil \\
\cline { 2 - 5 } & Fuerte & $\begin{array}{c}\text { Political implementation } \\
\text { (plan UCPN en Perú }\end{array}$ & $\begin{array}{c}\text { Symbolic implementation } \\
\text { (proyecto OLPC en Paraguay) }\end{array}$ & \\
\hline
\end{tabular}

Fuente: Elaboración propia.

Indudablemente, para implementar políticas de inclusión digital a gran escala, se necesita pericia no solo técnica (dominio de los TICs, conectividad, redes de usuarios, etc.), sino también diferenciada (soportes didácticos, capacitación, suply chain, compra masiva, etc.). En esta parte, sostenemos que un solo ministerio central -como nos lo enseñó el caso del Minedu en Perú, es incapaz de llevar a cabo un programa de acción pública tan transversal y exigente como los programas de inclusión digital. Para enfrentar todos los desafíos técnicos y logísticos que se plantean, un gobierno debe buscar sinergias interinstitucionales más allá de su administración central, apoyándose sobre conocimientos y competencias de otras instituciones como agencias, entes autónomos, empresas y hasta organizaciones de la sociedad civil. Asimismo, la gestión del plan Ceibal en Uruguay y sobre todo del plan CI en Argentina, constituyen pruebas concretas de esta búsqueda de la integración intersectorial. En otras palabras, a diferencia de Perú, Argentina y Uruguay supieron superar una gestión tradicional de tipo patrimonialista.

\section{Una gestión patrimonialista en Perú}

Para explicar por qué la gestión del plan UCPN ha sido tan caótica, invocamos dos factores de índole institucional: un problema de competencia burocrática y una lógica de funcionamiento autárquico, parasitario y muy poco proclive a la apertura hacia la sociedad civil.

En el Minedu, los funcionarios de base no son lo suficientemente competentes. En regla general, eran antiguos docentes que no tenían ninguna experiencia anterior en términos de gestión administrativa. Además, sus jefes, los altos funcionarios responsables de los programas educativos o de los diferentes departamentos internos, no gozaban tampoco de una gran legitimidad institucional por ser los correligionarios del ministro de turno. Como lo precisa Balarin (2005), las lógicas de cooptación políticas que imperan en la selección de los cuadros administrativos, son muy estériles a medio y largo plazo, ya que contribuyen solamente a formar "burocracia de pactos" (bureaucracy of pacts). Cuando el ministro de turno es revocado (en promedio una vez al año durante las presidencias de Fujimori y Toledo), se rompe el pacto-administrativo fijado con sus correligionarios 
alrededor de un eje, programa o proyecto político en particular (por ejemplo, el plan Huascarán en los años 2000).

En otras palabras, cuando el equipo de dirección es reemplazado por un nuevo equipo, se define un nuevo programa prioritario. Por consiguiente, no hay posibilidad de continuidad en términos de acción pública. El proyecto UCPN es revelador de esta burocracia de pacto. En efecto, Oscar Becerra, su responsable en el Minedu, era encargado de la dirección de la planificación en la Universidad San Martin de Porres (USMP), cuando el ministro Chang era rector de esta misma universidad. Con el cambio de gobierno y la llegada de un nuevo ministro de educación en julio de 2011, Oscar Becerra fue reemplazado por Luis Marcone, el cual había criticado en las columnas de un famoso periódico británico la inversión masiva en computadoras por la administración Chang ${ }^{7}$. Bajo la dirección de Marcone, se armaron nuevas prioridades en materia de inclusión digital, con más capacitación docente y contenidos pedagógicos. En consecuencia, el proyecto UCPN fue poco a poco abandonado.

Más allá del cambio recurrente de los cuadros administrativos que genera una importante inercia administrativa en el Minedu, el principal problema institucional consiste en la falta de cooperación interministerial. Como lo han demostrado Carmen Franco y Vázquez (2008), la gestión transversal de las políticas públicas en Perú es mínima o hasta imposible. No hay coordinación entre las diferentes administraciones, lo que ocasiona competición presupuestaria (ineficiencia), duplicación de programas (ineficacia) y distorsiones logísticas (incoherencia). En el caso del plan UCPN, este problema de insularidad interinstitucional (Waissbluth, 2003) se ejerce también dentro del propio ministerio, o sea se convierte en un problema de balcanización intrainstitucional.

En efecto, la gestión del plan UCPN ha sido confiada a un departamento ad hoc, creado a propósito y bautizado Dirección general de las tecnologías educativas (DIGETE). Fundado por decreto en junio $2007^{8}$, la DIGETE reunía una veintena de funcionarios. Dirigido por Oscar Becerra durante el mandato ministerial de Chang, la DIGETE se añadió a un organigrama ministerial ya muy fragmentado, con 12 departamentos de dirección y una quincena de oficinas suplementarias dependiendo, o de la secretaría general, o de la secretaría de planificación, las cuales son asociadas al gabinete del ministro. Ahora bien, como nos lo confió un funcionario del Minedu, la DIGETE funcionó de manera autárquica, en la medida en que no solicitó el apoyo de otras direcciones que hubieran podido ser útiles a la hora de implementar el plan UCPN, como por ejemplo la Dirección de la educación básica regular (para los contenidos pedagógicos) o la Dirección general de desarrollo docente (para los temas de capacitación).

"La DIGETE tenía una firme decisión política de implementar el programa. (...) Si no coordinábamos con ellos, igual se iba a hacer. Lo que hicimos fue tratar de manejarnos lo mejor posible enmarcados en el diseño que ellos tenían, para que tuviera cierto sustento pedagó- 
gico. Pero no fue un diseño institucional que fue acertado entre la DIGETE y nuestra dirección. Esto está clarísimo. La coordinación fue claramente insuficiente."

Además, como nos lo precisó también la responsable de otra dirección general, la DIGETE apareció también como una institución parasitaria, en el sentido de que su propósito (las tecnologías digitales) consistía en una temática transversal y no sectorial.

“Antes habían 5 direcciones generales (DIGETE incluido), hoy hay 8. Si en mi época, era difícil articular los proyectos de una dirección a otra - cada una hacia lo suyo, me imagino que debe ser peor ahora... (...) Yo hubiera ido al contrario en una mayor concentración, menos proliferación. Por ejemplo, la DIGETE. Para mi, no debería ser una dirección general. Para mi, es algo claramente transversal.” 10

Por añadidura, la DIGETE rechazó la mano tendida por las asociaciones de la sociedad civil que querían participar a la implementación del proyecto OLPC. Un miembro de la comunidad de los software libres nos aseguró que enviaron una carta a la DIGETE para proponer su ayuda, pero no obtuvieron respuesta. Oscar Becerra, el director reconoció que la cooperación con la sociedad civil fue insuficiente, justificándose por una sospecha de colaboración interesada.

"Lamentablemente, nos costo mucho integrarlos... Pues, si tuvimos el grupo de Escuelab, la gente de Linux, Sugar. (...) Pero hay que decir que mucha gente se suma a estas cosas, solamente para figurar. Es decir recibir invitaciones, irse de viaje... y no para realmente producir. Eso si nos faltó.” ${ }^{11}$

Como lo revela esta cita, la desconfianza por parte de la administración pública hacia la sociedad civil es muy grande. De hecho, la relación Estado/sociedad civil en Perú sigue siendo muy marcada por lógicas de confrontación (Grompone, 2004). En Argentina y Uruguay por lo contrario, la integración de la sociedad civil a la implementación de los planes Ceibal y CI fue muy alta.

\section{Participación ciudadana y cooperación interinstitucional en el estuario de la Plata}

En Uruguay, la institucionalización del plan Ceibal se puede explicar por la movilización ciudadana en su favor. Compuesta de varias centenas de miembros y presente en todo el territorio nacional, la Red de apoyo al plan Ceibal (Rap-Ceibal) se fundó pocas semanas después del lanzamiento del proyecto piloto de Cardal. Particularmente activo durante los dos primeros años, la Rap-Ceibal constituyó un movimiento social algo atípico, en la medida en que no tenía ninguna finalidad contestataria. Por lo contrario, reunió unos dos mil voluntarios (estudiantes, informáticos, ingenieros, etc.), 
solamente interesados por el éxito de la política de inclusión digital. Representaron diez veces el personal contratado por el Laboratorio tecnológico de Uruguay (LATU). Cualificada de "inestimable" por una formadora en tecnología numérica (Rabajoli, 2009, p.77), la ayuda de los voluntarios fue particularmente importante durante la fase de universalización del plan Ceibal, entre mayo 2007 y octubre 2009. Bajo la coordinación del LATU, participaron a la distribución de las computadoras, a la capacitación de los docentes, a la realización de las cartografías numéricas necesarias, a la instalación de las antenas internet en las escuelas, y hasta a la concepción de herramientas didácticas informáticas.

Del mismo modo, en Argentina, una asociación de militantes para la promoción de software libres se aproximó de ANSES con la ambición de crear una interface virtual bautizada Huayra - lo que significa "viento" en quechua. Iniciado en el 2011, el proyecto Huayra dio una nueva trayectoria al plan CI. En efecto, desembocó en la creación del primer software libre específicamente argentino. A partir de 2013, además de Microsoft el sistema operativo Huayra equipaba todas las computadoras del plan CI, bajo el principio del Dual-Boot. Recuperando el discurso antiimperialista de la presidenta, el objetivo de los partidarios del software libres era de desestimular la juventud argentina con el uso de tecnologías numéricas privadas norteamericanas. Sus líderes fueron contratados por ANSES e incorporados en el Centro nacional de investigación y de desarrollo de tecnologías libres (Cenital). In fine, mientras que el caso del Rap-Ceibal es representativo de las relaciones bastante harmoniosas que unen la izquierda uruguaya con las organizaciones de la sociedad civil (Rossel, 2013), el caso del proyecto Huayra revela, del mismo modo, las estrategias de cooptación (IppolitoO’Donnell, 2013), que son también muy típicas del gobierno de los Kirchner vis a vis los movimientos sociales.

No obstante, más allá de la integración de las asociaciones civiles al proceso de implementación, la institucionalización de los programas de inclusión digital en Uruguay y Argentina, se explica por sus arquitecturas institucionales respectivas. En ambos casos, el gobierno movilizó las energías, conocimientos y competencias de varias instituciones. Mientras que en Perú solo participó a la gestión logística del plan UCPN un solo departamento del Minedu, en Uruguay y Argentina, participaron varios organismos, tanto públicos (ministerios), como parapúblicos (agencias, entes autónomos) y hasta empresas privadas. Asimismo, aunque en la primera fase del plan Ceibal (distribución de computadoras) se privilegió una lógica by-pass (Midaglia, 1998), o sea de contorneo administrativo en contra de las instituciones educativas inicialmente opuestos al proyecto (Larrouqué, 2013), intervinieron finalmente en la gestión e implementación de plan Ceibal por lo menos seis instituciones, las cuales conformaron, por decreto ${ }^{12}$, la Comisión de políticas: el LATU (organismo gestor), la empresa de telecomunicación Antel (conectividad), el MEC y la ANEP (capacitación y contenido pedagógico), y dos agencias (la AGESIC y ANNI) vinculadas con las innovaciones numéricas (soporte técnico). 
Esta lógica de cooperación intersectorial fue aún más relevante en Argentina. De hecho, el decreto del plan CI fundó expresamente un Comité Ejecutivo interinstitucional ${ }^{13}$, compuestos por representantes de ANSES (organismo gestor), del Instituto nacional de formación docente (capacitación), de Educ.ar (contenido pedagógico) - ambos dependiendo del ministerio de Educación de la Nación, del subsecretario a las tecnologías de gestión que depende de la Jefatura de Gabinete de Ministros (seguridad informática) y de la secretaría de comunicación (SECOM) que depende del ministerio de planificación (conectividad). A esta estructura directiva, se añaden otros organismos que intervinieron de manera puntual en la implementación del plan CI como por ejemplo Correo Argentinos y OCA para la distribución de computadoras o empresas de telecomunicaciones privadas (Telefónica, Claro, Telecom, Telmex, etc.) para suplir el proveedor satelital público Arsat. Esta colaboración interinstitucional es inédita en un país donde las rivalidades institucionales se vivían como rivalidades deportivas fratricidas (Acuña, 2001) ${ }^{14}$.

\section{Conclusiones: Reconsiderar el liderazgo presidencial}

En este artículo intentamos revelar los factores de la institucionalización de las políticas de inclusión digital, al comparar el proyecto UCPN en Perú, abandonado a mediados del año 2011, con los planes Ceibal y CI, todavía vigentes en Uruguay y Argentina. Una vez descartada la hipótesis de una posible influencia nefasta del contexto de la decisión en el destino de la política pública, sostuvimos que la calidad del policy design es determinante, o sea se necesita precisión y esmero al nivel de la preparación logística. A diferencia de los gobiernos del estuario de la Plata, la elite político-administrativa de Perú no consagró la atención suficiente a esta etapa matricial del proceso de implementación. Como lo demostramos, la preparación logística del plan UCPN ha sido grandemente improvisada, lo que perjudicó su ejecución administrativa.

Más allá de la coherencia del policy design, recalcamos en nuestra segunda parte la importancia de la innovación institucional. Uruguay y Argentina lograron impulsar nuevas modalidades de gestión pública al favorecer, no solamente, la participación de la sociedad civil sino también, la cooperación interinstitucional. En este sentido, mientras que la implementación del plan UCPN fue tradicional, verticalista, y hasta muy patrimonialista, la de los planes Ceibal y CI fue bastante revolucionaria. En breve, podríamos decir que los programas de inclusión digital de Uruguay y Argentina no fueron vanguardistas en el fondo (lucha contra la brecha digital), sino también en la forma (lógicas de integración intersectorial). De manera general, esas políticas públicas son hasta reveladoras del "regreso del Estado" en materia educativa y social. En otras palabras, ilustran el paradigma posneoliberal, tal cual se desarrolló en el Cono Sur (García Delgado, 2014).

Añadiremos que el liderazgo presidencial parece haber sido igualmente muy importante en la institucionalización de las políticas de inclu- 
sión digital. Sin dejarse enceguecer por el "voluntarismo político" o el "fetichismo de la decisión” (Lascoumes y Le Galès, 2009, p.16), no se puede tampoco perder de vista el rol del presidente en la conducta de la acción pública. Como lo demostró Cohen (1997), es mejor para un presidente ser audaz en sus resoluciones políticas que simplemente receptivo (responsive) vis $a$ vis las expectativas de sus conciudadanos. Como la opinión pública es muy versátil, tomar simplemente en consideración las expectativas ciudadanas le da solamente al presidente un respaldo de corto plazo. Si quiere obtener un apoyo más largo e idealmente marcar su época, tiene que ser capaz de implementar políticas públicas efectivas. Asimismo, tiene que afirmar su liderazgo todo a lo largo del policy making process, desde la puesta en la agenda hasta la despliegue territorial. En el caso de Uruguay y Argentina, tenemos dos ejemplos fehacientes de una firme implicación presidencial todo a lo largo del proceso de implementación del plan Ceibal y CI.

A diferencia de Alan García - que se mostró mucho más proclive a exponer a su ministro de Educación, Tabaré Vázquez y Cristina Kirchner no vacilaron en asociar plenamente su imagen con el plan Ceibal y CI. Con la voluntad de revalorizar el sistema público de enseñanza, cada uno asumió un rol de protagonista clave. En su discurso de lanzamiento respectivo, ambos usaron de los mismos campos semánticos (justicia social e innovación tecnológica) y se inscribieron en una tradición de excelencia escolar equivalente. Vázquez rememoró la gloriosa “revolución vareliana de 1875”15. En cuanto a Cristina Kirchner, en plena conmemoración del bicentenario de la Independencia, confesó "sentirse un poco la Sarmiento del siglo XXI"16. En el caso de Perú y con la excepción del prefacio del manual de uso del XO de unas 6 líneas a penas ${ }^{17}$, no se encontró ningún marco de involucramiento de Alan García con el plan UCPN. Como si hubiera buscado "evitar la condenación” (blame avoidance, Caune, 2010), Alan García no quiso asumir la responsabilidad política del proyecto OLPC. La dejó a su ministro Chang que le sometió la iniciativa. Este la hizo recaer sobre su colega de la USMP Oscar Becerra, el cual tuvo que enfrentar todas las críticas.

En Perú, se constató un problema de falta de liderazgo y de dilución de la autoridad que perjudicó la coherencia del programa. Al contrario en el estuario de la Plata, los dos presidentes metieron en juego su propia credibilidad política, en caso de fracaso de las políticas de inclusión digital. Al cristalizar los desafíos logísticos así como las expectativas de los jóvenes, los planes

Ceibal y CI se convirtieron en prioridades nacionales, imponiéndose en las agendas política, administrativa y electoral. Así, cuando el presidente Vázquez anuncia, unos diez días antes de Navidad, que "Uruguay va a ser el primer país en América en cubrir [en herramientas informáticas] a todos los niños en edad escolar"18, lanza a todas las instituciones involucrada y a las fuerzas vivas de la Nación un inmenso y muy arriesgado desafío. Está consciente que no tiene derecho al error.

Del mismo modo, cuando la presidenta Kirchner sueña públicamente con que "todos nuestros estudiantes secundarios de la escuela pública 
puedan en el lapso que va del año 2010 hasta el 2012, en un programa de casi 3 años, contar con su netbook"19 , erige encima de su propio gobierno una espada de Damocles. Al suscitar tanto entusiasmo por parte de la juventud, esas políticas públicas no toleran el fracaso. Determinados en asumir un desafío que se impusieron ellos-mismos, los dos líderes del estuario de la Plata pusieron una enorme presión sobre sus administraciones. De este modo, contribuyeron a galvanizar las energías institucionales a favor del éxito de estos programas e hicieron posibles las innovaciones políticoadministrativas (estrategia by pass e integración intersectorial). Al contrario, en el caso peruano, la pasividad relativa de Alan García deja pensar que el líder aprista haya anticipado el atolladero administrativo en el cual se iba a sumergir el proyecto UCPN. 


\section{Notas}

${ }^{1}$ Entre 2007 y 2013, el gobierno uruguayo distribuyó más de un millón de laptops, o sea triplicó la cifra respecto a los 350000 alumnos inicialmente considerados por el programa. El plan Ceibal se inscribió en la duración, festejando su décimo cumpleaños en el 2017. En Argentina, entre 2010 y 2016, fueron entregadas a los alumnos del secundario más de 5600000 computadoras, lo que representa dos veces el objetivo inicial. En 2013, el costo total había alcanzado casi los 4000 millones de dólares. Cf. Artículo de El Observador del 7 de octubre 2013 titulado "Se entregó la ceibalita un millón” y artículo de El Auditor info del 17 de julio de 2013, titulado: "El presupuesto para el programa de las netbooks aumentó un $130 \%$ \%.

${ }^{2}$ Cf. Comentarios de los lectores relativos a los artículos del Educational Technology Debate titulados " OLPC in Peru: A Problematic Una Laptop Por Niño Program » y " Oscar Becerra on OLPC Peru's Long-Term Impact ", respectivamente publicados en el sitio web del think tank norteamericano el 27 de octubre 2010 et el 13 de marzo de 2012.

${ }^{3}$ Cf. artículo de La República publicado el 14 de octubre 2012 y titulado « Robots comprados por el regimen aprista se habrian sobrevalorado ".

${ }^{4}$ Cf. artículos de La Nación de 8?de abril, del 10 y 13 de mayo 2010, respectivamente titulados: " La promesa de las computadoras ", "Lanzarán planes para atraer votos de la clase media » $\mathrm{y}$ " Sobornar al que vota ».

5 Se entiende por "elite programática” un grupo de actores fuertemente institucionalizados, especializados en un sector de intervención estatal (lo social, esencialmente) y que comparten los mismos diagnósticos y soluciones en materia de acción pública. Al haber adquirido conocimientos específicos y saberes técnicos precisos, tienen capacidad de innovación. Asimismo, pueden aconsejar los hombres políticos sobre nuevos instrumentos de políticas públicas.

${ }^{6}$ Esta prórroga se explica por la voluntad del gobierno argentino de testear, antes de una eventual extensión nacional, varios modelos de laptops a micro-escala y en función de diferentes públicos escolares. Como los XO de la fundación OLPC no se revelaron conformes con las expectativas, se decidió adoptar las computadoras Classmate producidas por la multinacional Intel y dirigidas a los alumnos de secundaria (Diaz Rato y Fontdevlia, 2009).

${ }^{7}$ Cf. Artículo de The Economist publicado el 7 de abril 2012 y titulado : « Education in Peru : Error Message. A disappointing return from an investment in computing ».

${ }^{8}$ Cf. Artículo 1 del Decreto Supremo n016-2007-ED

${ }^{9}$ Cf. Entrevista anónima. Minedu. Marzo 2013.

${ }^{10}$ Cf. Entrevista con Miriam Ponce Véritz, ex-responsable de la Dirección de la educación básica regular, Minedu, 21 de marzo 2013.

${ }^{11}$ Cf. Entrevista con Oscar Becerra, ex-responsable de la DIGETE, en su oficina de la USMP, el 26 de marzo 2013. 
Polis, Revista Latinoamericana, $N^{\circ} 48,2017$

${ }^{12}$ Cf. Artículo 2 del Decreto EC/579.

${ }^{13}$ Cf. Artículo 2 del Decreto 459/10.

${ }^{14}$ Asímismo, Acuña (2001) compara la oposición entre los ministerios al mítico derby de futbol entre Boca Junior y River Plate!

${ }^{15}$ Extracto del discurso del presidente de la República Oriental del Uruguay, pronunciado el 14 de diciembre de 2006.

${ }^{16}$ Extracto del discurso de la presidente de la República Argentina, pronunciado el 6 de abril de 2010.

${ }^{17}$ Digete. Manual del Docente para el uso de la laptop XO. Lima, Perú : Minedu, 2008, p. 3.

${ }^{18}$ Extracto del discurso del presidente de la República Oriental del Uruguay, pronunciado el 14 de diciembre de 2006.

${ }^{19}$ Extracto del discurso de la presidenta de la República Argentina, pronunciado el 6 de abril de 2010. 


\section{Bibliografía}

Acuña, C. (2001). Problemas político-institucionales que obstaculizan mejores políticas públicas en la Argentina de hoy. Revista del CLAD Reforma y Democracia, 19, 1-10.

Agosta, L. et al. (2012). Mejorar la calidad de las políticas públicas para restaurar la confianza en el gobierno, Revista del CLAD Reforma y Democracia, 52, 83-104

Barboza Norbis, L. (2012). Origen y evaluación del Plan Ceibal. Estudio exploratorio del modelo educativo subyacente (2007-2011), Cuadernos de Educación de la UDE, 1-75.

Balarin, M. (2005). Radical discontinuity. A study of the role of education in the Peruvian state and of the institutions and cultures of policy making in education (tesis doctoral). University of Bath, Bath, Inglaterra.

Balarin, M. (2013). Las políticas TIC en los sistemas educativos de América Latina. Caso Perú. Buenos Aires, Argentina : Unicef.

Caune, H. (2010). Blame avoidance (évitement du blâme). En L. Boussaguet, et al (eds). Dictionnaire des politiques publiques (pp.114-123). París, Francia : Presses de Sciences Po.

Cunill Grau, N. (2014a). La intersectorialidad en las nuevas políticas sociales. Un acercamiento analítico-conceptual. Gestion y Política Pública, 23 (1), 5-46.

Cunill Grau, N. (2014b). Resistencias a la colaboración interinstitucional. Aprendizajes para la implementación de las nuevas políticas sociales. Documentos y Aportes en Administración Pública y Gestión Estatal, 14 (13), 7-32.

Cristia J. et al. (2012). Tecnología y desarrollo en la niñez?: evidencia del programa Una Laptop por Niño. Washington DC, Estados-Unidos : BID.

Cohen, J. (1997). Presidential Responsiveness and Public Policy-Making : The Public and the Policies that President Choose. Ann Arbor, Estados Unidos : The University of Michigan Press.

Comisión de políticas, (2009). El plan Ceibal. Breve descripción y principales líneas de acción. En Unesco, En el camino del Plan CEIBAL (pp.29-39). Montevideo, Uruguay: Unesco.

De Luca, M. y Malamud, A. (2010). Argentina : turbulencia económica, polarización social y realineamiento político. Revista de Ciencia Política, 30 (2), 173-189. 
Del Carmen Franco, M. y Vásquez, E. (2008). La (in)viabilidad de la fusión de los programas sociales en el Perú. Apuntes, 62, 277-124.

Diaz Rato, S. Fontdevlia, P. (2012). Inclusión digital y calidad educativa : el Programa Conectar Igualdad entre 2010 y 2011, Buenos Aires : Argentina, Eduntref.

Draibe, S. y Riesco, M. (2007). Latin America: A New-Developmental Welfare State in the Making? En M. Riesco (coord.), Latin America. A New-Developmental Welfare State Model in the Making? (pp.21113), Nueva York, Estados Unidos: Palgrave Macmillan.

Dubois, V. (2010). Politiques au guichet, politique du guichet. En O. Borraz y V. Guiradon (Eds.), Politiques Publiques. 2. Changer la société (pp.265-286). París, Francía : Presses de Sciences Po.

Fernández Toro, J. (2009). Los desafíos contemporáneos del Estado. Aportes para el Estado y la administración gubernamental, 27, 135-152.

Genieys, W. (2007). L'émergence d'élite(s) programmatique(s) face à la mutation de l'État français, Working paper de l'Institut de Ciències Polítiques i socials, 261, 1-28.

Grompone, R. (2004). Posibilidades y límites de experiencias de promoción de la participación ciudadana en el Perú. Documento de Trabajo IEP, 132, 1-46.

Hill, M. y. Hupe, P (2014). Implementing Public Policy. An Introduction to the Study of Operational Governance. London, Inglaterra: Sage.

Ippolito-O’Donnel, G. (2013). Calidad institucional y sociedad civil en la Argentina. En Acuña, C. (eds.) ¿ Cuánto importan las instituciones ? Gobierno, Estado y actores en la política argentina (pp. 325-363). Buenos Aires, Argentina : Siglo XXI Editores.

Jobert, B. y Muller, P. (1987). L'État en action. Politiques publiques et corporatismes. París, Francia, PUF.

Jones, C. (1984). An Introduction to the Study of Public Policy. Third Edition. Monterey, Estados Unidos: Brooks/Cole Publishing.

García Delgado, D. La provisión de bienes y servicios públicos en los gobiernos progresistas del Cono Sur. La década ganada (2002-2013). Revista Estado y Políticas Públicas, 2, 20-46.

Kingdon, J. (1984). Agenda, Alternatives and Public Policies. Nueva York, Estados Unidos: Harper Collins.

Lago Martínez, S. et al. (2012). Inclusión digital en la educación pública 
argentina. El programa Conectar Igualdad. Revista Educación y Pedagogía, 24 (62), 205-218.

Lanzaro, J. (2010). Uruguay : un gobierno social democrático en América Latina. Revista Uruguaya de Ciencia Política, 19 (1), 45-67.

Larrouqué, D. (2013). La implementación del plan Ceibal : Coaliciones de causa y nueva gerencia pública en Uruguay. Revista Uruguaya de Ciencia Política, 27 (1), 37-58.

Larrouqué, D. (2016). Les politiques d'inclusion digitale en Amérique latine: de la rénovation éducative à la recomposition de l'Etat (Argentine, Paraguay, Pérou, Uruguay) (Tesis doctoral). Sciences Po, París, Francia.

Larrouqué, D. (2017a). Etat de grâce et fenêtre d’opportunité politique : les victoires historiques de Vázquez (Uruguay) et Lugo (Paraguay) en perspective comparée. Nuevo Mundo Mundos Nuevos, Extraits de thèses.

Larrouqué, D. (2017b). Le moment de la décision politique est il important? Une comparaison du contexte de mise en œuvre des programmes d'inclusion digitale au Pérou et en Argentine. Working Paper de l’Opalc, septembre 2017.

Lascoumes, P. y Le Galès, P. (2009). Sociologie de l'action publique. París, Francia : Armand Colin.

Laura Quispe, C. (2015). Maestros y computadoras portatiles en el Perú : ¿ por qué no se usan las computadoras portátiles ?, Apertura, 7 (1), 118.

Lipsky, M. (2010). Street level bureaucracy. Dilemmas of the Individual in Public Services (1980). Nueva York, Estados-Unidos: Russell Stage Foundation.

Maillet, A. (2012). Beyond the Minimal State: Sketching an Alternative Agenda. Revista de Ciencia Política, 32 (3), 687-701.

Matland, R. (1995). Synthesizing the Implementation Literature : The Ambiguity-Conflict Model of Policy Implementation. Journal of Public Administration Research and Theory, 5 (2), 145-174.

Mazmanian D. y Sabatier, P (1983). Implementation and Public Policy. Glenview, Estados Unidos: Scott, Foresman and company.

May P. (2003). Policy Design and Implementation. En G. Peter y J. Pierre (Ed.), Handbook of Public Administration (pp.223-234). London, Inglaterra: Sage. 
Nef, J. (2003). Public Administration and Public Sector Reform in Latin America. En G. Peter y J. Pierre (Ed.), Handbook of Public Administration (pp.523-536). London, Inglaterra: Sage.

Oliart, P. (2011). Mediocridad y corrupción : los enemigos de la educación pública. En L. Pásara (Ed.) Perú ante los desafíos del siglo XXI (pp. 295-325) Lima, Perú : Fondo Editorial PUCP.

Oppenheimer, A. (2010). i Basta de historias ! La obsesión latinoamericana con el pasado y las 12 claves del futuro. Montevideo, Uruguay : Debate.

Pressman, J. y Wildavsky A. (1984). Implementation [1973]. Berkeley, Estados Unidos : University of California Press.

Quispe, C. y Díaz, E. (2009). Una Laptop Por Niño en escuelas rurales del Perú : un análisis de lasbarreras y facilitadores. Lima, Perú : CIES.

Rabajoli, G. (2009). Origen y desafíos de una estrategia de inclusión : el Plan Ceibal. En Unesco. En el camino del Plan CEIBAL (pp.70-84). Montevideo, Uruguay : Unesco.

Ravinet, P. (2010). Fenêtre d'opportunité. En L. Boussaguet, et al (eds). Dictionnaire des politiques publiques (pp.274-282). París, Francia : Presses de Sciences Po.

Rivas, A. et al. (2004). Gobernar la educación. Estudio comparado sobre el poder y la educación en las provincias argentinas. Buenos Aires, Argentina: Granica.

Rivoir, A. L., Lamschtein, S. (2012), Cinco años del Plan Ceibal. Algo más que una computadora para cada niño. Montediveo, Uruguay: Unicef.

Rodriguez, A. (2010). La redefinición del Estado y su rol. Aportes para el Estado y la administración gubernamental, 28, 31-40.

Romero Quintero, B. (2013). Las políticas públicas como muestra de la gestión de un Estado, Pensamiento Americano, 6 (11), 114-121.

Rossel, C. (2013). «Te amo, te odio, dame más ». ONG, participación y representación en el primer gobierno de izquierda en Uruguay. Buenos Aires, Argentina : CLASCO.

Savard, J.F. (2010). La cohérence des politiques publiques. En S. Paquin et al. (Eds). L'analyse des politiques publiques (pp. 309-335). Montreal, Canada : Les Presses Universitaires de Montréal. 
Schweinheim, G. (2010). Estado, administración y desarrollo. Contribución a un paradigma de investigación y políticas estatales para un nuevo desarrollo en América latina. Aportes para el Estado y la administración gubernamental, 28, 101-136.

Thwaites Rey, M. (2008). ¿Qué Estado tras el experimento neoliberal? Revista del CLAD Reforma y Democracia, 41, 33-56.

Vallespín, F. (2010). La transformación del Estado como consecuencia de los impactos de la crisis económica. Revista del CLAD Reforma y Democracia, 48, 31-50.

Villanueva, E., et al. (2011). Nuevas voces, nuevos escenarios?: estudios evaluativos sobre el programa Conectar Igualdad. Buenos Aires, Argentina, Ministerio de Educación.

Waissbluth, M. (2003). La insularidad de la gestión pública latinoamericana. Revista del CLAD Reforma y Democracia, 27, 1-7

Recibido: 05.09.17

Aceptado: 03.11.17 